\title{
OH maser towards IRAS 06056+2131: polarization parameters and evolution status
}

DOI:

10.1093/mnras/staa2810

\section{Document Version}

Accepted author manuscript

Link to publication record in Manchester Research Explorer

\section{Citation for published version (APA):}

Darwish, M. S., Richards, A. M. S., Etoka, S., Edris, K., Saad, S., Beheary, M., \& Fullar, G. (2020). OH maser towards IRAS 06056+2131: polarization parameters and evolution status. Royal Astronomical Society. Monthly Notices, 499(1), 1441-1449. https://doi.org/10.1093/mnras/staa2810

\section{Published in:}

Royal Astronomical Society. Monthly Notices

\section{Citing this paper}

Please note that where the full-text provided on Manchester Research Explorer is the Author Accepted Manuscript or Proof version this may differ from the final Published version. If citing, it is advised that you check and use the publisher's definitive version.

\section{General rights}

Copyright and moral rights for the publications made accessible in the Research Explorer are retained by the authors and/or other copyright owners and it is a condition of accessing publications that users recognise and abide by the legal requirements associated with these rights.

\section{Takedown policy}

If you believe that this document breaches copyright please refer to the University of Manchester's Takedown Procedures [http://man.ac.uk/04Y6Bo] or contact uml.scholarlycommunications@manchester.ac.uk providing relevant details, so we can investigate your claim.

\section{OPEN ACCESS}




\title{
OH maser toward IRAS 06056+2131: polarization parameters and evolution status
}

\author{
Darwish, M. S. ${ }^{1,2},{ }^{\star}$ Richards, A. M. S. ${ }^{3}$, Etoka, S. ${ }^{3}$, Edris, K. A. ${ }^{4}$, \\ Saad, S. M. ${ }^{1,2}$, Beheary, M. M. ${ }^{4}$, Fuller, G. A. ${ }^{3}$ \\ ${ }^{1}$ Astronomy Department, National Research Institute of Astronomy and Geophysics (NRIAG), 11421 Helwan, Cairo, Egypt. \\ ${ }^{2}$ Kottamia Center of Scientific Excellence in Astronomy and Space Science (KCScE, STDF No. 5217, ASRT), Cairo, Egypt. \\ ${ }^{3}$ Jodrell Bank Centre for Astrophysics, Department of Physics \& Astronomy, The University of Manchester, M13 9PL, UK. \\ ${ }^{4}$ Astronomy and Meteorology Department, Faculty of Science, Al-Azhar University, Cairo, Egypt.
}

Accepted 2020 September 11. Received 2020 September 9; in original form 2020 July 22

\begin{abstract}
We present high angular resolution observations of $\mathrm{OH}$ maser emission towards the high-mass star forming region IRAS $06056+2131$. The observations were carried out using the UK radio interferometer array, Multi-Element Radio Linked Interferometer Network (MERLIN) in the $\mathrm{OH}$ main lines at 1665- and 1667-MHz, in addition to the $\mathrm{OH}$ satellite line at $1720-\mathrm{MHz}$. The results of this study reveal the small upper limit to the size of emission in the $1665-\mathrm{MHz}$ line with an estimated total intensity of $\sim 4 \mathrm{Jy}$. We did not detect any emission from the 1667-MHz and $1720-\mathrm{MHz}$ lines. The full polarization mode of MERLIN enables us to investigate the magnetic field in the $\mathrm{OH}$ maser region. In this transition, a Zeeman pair is identified from which a magnetic strength of $\sim-1.5 \mathrm{mG}$ is inferred. Our results show that IRAS $06056+2131$ is highly polarized, with $\sim 96 \%$ circular polarization and $\sim 6 \%$ linear polarization. The linear polarization angle is $\sim 29^{\circ}$, implying a magnetic field which could be aligned with the outflow direction detected toward this region, but the actual magnetic field direction has an uncertainty of up to $110^{\circ}$ due to the possible effects of Faraday rotation. The star forming evolutionary status of the embedded proto-stellar object is discussed.
\end{abstract}

Key words: Stars: formation - stars: massive - stars: individual: IRAS 06056+2131 - masers - Polarization

\section{INTRODUCTION}

Despite the important role that the high-mass stars play in the formation of stars and galaxies as well as the evolution of the universe, many questions are still open in this issue. Different theoretical approaches have been introduced in order to answer the question of how massive stars form. One approach, proposed by McKee \& Tan (2003), is known as the core accretion model. They suggested that the formation of massive stars is similar to that of low mass stars, where the dominant force is the magnetic field and sufficient mass accretion only occurs when the magnetic support is removed through jets and outflows (e.g. Mouschovias \& Paleologou 1979; Mouschovias et al. 2006; Commerçon et al. 2011; Tan et al. 2013; Klassen et al. 2017).

On the other hand, other authors (e.g. Padoan \& Nordlund 2002; Mac Low \& Klessen 2004; Kitsionas et al. 2005; Vázquez-Semadeni et al. 2011) argue that dynamical influences such as turbulence play more effective roles than the magnetic field, particularly in the early stages of massive star formation. Therefore, understanding the role of the magnetic

\footnotetext{
* E-mail: darwish.msk@gmail.com
}

field during the formation of massive stars can lead us to a better understanding of how such stars are formed.

Maser emission lines provide exceptionally high resolution probes to measure the small scale magnetic field strength and structure within 10s - 1000s AU of high mass protostars (e.g. Fish \& Reid 2007; Vlemmings et al. 2010; Surcis et al. 2013; Goddi et al. 2017; Crutcher \& Kemball 2019). Masers are also used to investigate the kinematics as well as the physical conditions surrounding massive protostellar objects from the onset of formation (e.g. Caswell 1997; Stahler et al. 2000; Szymczak \& Gérard 2004; Breen et al. 2010b; Darwish et al. 2020). Hydroxyl $(\mathrm{OH})$, water $\left(\mathrm{H}_{2} \mathrm{O}\right)$ and methanol $\left(\mathrm{CH}_{3} \mathrm{OH}\right)$ masers are commonly used to investigate the kinematics as well as the physical conditions surrounding massive protostellar objects in the early stage(s) of their formation. Due to its paramagnetic nature, the $\mathrm{OH}$ radical is consider to be more sensitive than $\mathrm{CH}_{3} \mathrm{OH}$ and $\mathrm{H}_{2} \mathrm{O}$ for measuring the Zeeman effect directly and consequently measuring the magnetic fields strength toward these objects (e.g. Cohen 1985; Edris et al. 2005; Green et al. 2007; Green et al. 2012; Vlemmings 2007; Etoka et al. 2012; Edris et al. 2017). However, $\mathrm{H}_{2} \mathrm{O}$ and $\mathrm{CH}_{3} \mathrm{OH}$ are also important particularly in studying the morphology of the magnetic field since their linear polarization vectors are less affected by Faraday rotation than $\mathrm{OH}$ masers 
(Vlemmings et al. 2006b; Surcis et al. 2011a,b; Momjian \& Sarma 2017). Zeeman splitting of $\mathrm{OH}$ maser lines can provide the $3 \mathrm{D}$ orientation of magnetic fields towards massive protostellar objects. The compactness and brightness of masers allow polarization observations at high angular resolution using interferometers, such as e-MERLIN, the VLBA (Very Long Baseline Array) and the EVN (European VLBI Network).

$\mathrm{OH}$ masers, particularly the main lines at 1665 and 1667 $\mathrm{MHz}$, are known to be frequently associated with different evolutionary stages of high-mass star forming regions (HMSFRs) (e.g. Ellingsen et al. 2007). Caswell et al. (2011) reported that $\mathrm{OH}$ masers are associated with Ultra-Compact H II (UC H II) regions (see also, Cohen et al. 1988; Braz et al. 1990; Breen et al. 2010a), other authors (e.g. Breen et al. 2010b; Edris et al. 2007; Garay \& Lizano 1999) found that $\mathrm{OH}$ masers can also be associated with HII regions, which represent a more advanced stage in the massive star formation time scale.

IRAS 06056+2131 (also known as AFGL6366S) is a high mass star-forming region located in the Gemini OB1 cloud, which is known to be one of the most massive molecular cloud complexes in the outer Galaxy. It is named after the IRAS source located at RA $(2000)=06^{h} 08^{m} 40^{s} .9$, Dec $(2000)=$ $21^{\circ} 31^{\prime} 00^{\prime \prime}$ with an error ellipse of $30 \times 5$ arcsec at a position angle (PA) of $91^{\circ}$ (Joint IRAS Science Working Group 1988), giving an uncertainty of $2.15,5 . \prime 0$ in RA and Dec, respectively.

This source is one of a large sample of candidate high-mass young stellar objects (YSO) which were identified by Palla et al. (1991). The sample of 260 IRAS sources was divided into two subsamples based on their IRAS color. The first subsample, so-called "high", is composed of sources where $[25 \mu \mathrm{m}$ $-12 \mu \mathrm{m}]>0.57^{1}$, which fulfils Wood \& Churchwell (1989) criteria for objects associated with ultra-compact H II (UC H II) regions. The second subsample, so-called "low", is composed of sources with $[60 \mu \mathrm{m}-12 \mu \mathrm{m}]>1.3$, where different evolutionary stages can be found, extending from the stage prior to the UCHII detection to evolved sources (Molinari et al. 1996). According to Palla et al. (1991), IRAS 06056+2131 belongs to the "high" subsample. The estimated far-infrared luminosity of IRAS $06056+2131$ is $5.83 \times 10^{3} L_{\odot}$ (Yoo et al. 2018 , and references therein) while the distance to the source is thought to be in the range of 0.8 to $2.5 \mathrm{kpc}$ (Koempe et al. 1989; Kurtz et al. 1994). We adopt a distance of $1.5 \mathrm{kpc}$ as an average (Zhang et al. 2005). A CO ( J=2-1) bipolar outflow was detected toward IRAS $06056+2131$ by Snell et al. (1988); Wu et al. (2004) and Zhang et al. (2005). Kurtz et al. (1994), using the VLA at a resolution $\leq 1^{\prime}$ detected weak radio continuum flux (less than $1 \mathrm{mJy}$ ) at $3.6 \mathrm{~cm}$, within $8 . .0$ of the IRAS source, while Rosolowsky et al. (2010), through the Bolocam Galactic Plane Survey (BGPS), indicated that IRAS $06056+2131$ is associated with $\mathrm{mm}$ continuum emission.

IRAS $06056+2131$ is known to be associated with several maser species. A Class II 6.7-GHz methanol maser was first detected toward the source at $12.7 \mathrm{Jy}$ and found to be offset from the nominal IRAS position by 7. .' 6 (see, Caswell et al. 1995; Szymczak et al. 2000; Xu et al. 2009; Fontani

$1\left[\lambda_{2}-\lambda_{1}\right]$ is defined as $\log _{10}\left[F_{\lambda 2} / F_{\lambda 1}\right]$, where $F_{\lambda i}$ is the IRAS flux density in wavelength band $\lambda_{i}$. et al. 2010). Water maser emission at 22-GHz was detected by Koempe et al. (1989) and Sunada et al. (2007) with peak flux densities of $23 \mathrm{Jy}$ and $2.43 \mathrm{Jy}$, respectively, although Palla et al. (1991) failed to detect any emission in this transition, suggesting that the water masers toward this source are variable. The first detection of the $\mathrm{OH}$ maser toward IRAS $06056+2131$ was reported by Turner (1979), while the first positional association was made by Cohen et al. (1988). The LSR (Local Standard of Rest) velocity for IRAS 06056+2131 given in SIMBAD is $2.5 \pm 1.6 \mathrm{~km} \mathrm{~s}^{-1}$, measured using SEST (Swedish-ESO Submillimeter Telescope) observations of CS by Bronfman et al. (1996) but there may be multiple sources within their $50^{\prime \prime}$ beam.

In this study, we aim to estimate the accurate position of the $\mathrm{OH}$ maser within the IRAS $06056+2131$ region and contribute to a better understanding of the evolutionary status of the IRAS source. Additionally, we investigate the magnetic field in the maser region using full-polarization MERLIN observations. In Section 2, the observations and data reduction are described. The data analysis and results of the imaging are given in Section 3. Our discussion and conclusion are presented in Section 4 and 5, respectively.

\section{OBSERVATIONS AND DATA REDUCTION}

The observations of the source IRAS $06056+2131$ were carried out using the MERLIN interferometer. Observations of the $\mathrm{OH}$ maser emission were performed in full-polarization mode during February 2007. IRAS 06056+2131 was observed for 3 full tracks, switching between $1665 \mathrm{MHz}$ and $1667 \mathrm{MHz}$ in addition to $1720 \mathrm{MHz}$ transitions about every 30 minutes during the observations.

The target was observed in bands centred on 1665.402, 1667.357 and $1720.520 \mathrm{MHz}$, adjusted to fixed velocity with respect to the LSR in the correlator assuming a target velocity of $10 \mathrm{~km} \mathrm{~s}^{-1}$ (Edris et al. 2007). The compact quasar $0617+210$ was observed as a phase reference calibrator with position $\mathrm{RA}=06^{h} 20^{m} 19^{s} .529$, Dec $=21^{\circ} 02^{\prime} 29^{\prime \prime} .501$. This was observed alternately with the target in an approximately 10 minute cycle, giving a total of about $6 \mathrm{hr}$ useful data ontarget at each frequency. $0617+210$ was observed in the full $16 \mathrm{MHz}$ bandwidth ('wide', $13 \mathrm{MHz}$ useful) with two tunings, one covering 1665 and $1667 \mathrm{MHz}$ and the other covering 1720 $\mathrm{MHz}$.

The data were extracted from the MERLIN archive and converted to FITS at Jodrell Bank Center for Astrophysics (JBCA) using local software (dprogs) (Diamond et al. 2003) and the Astronomical Image Processing System (AIPS) software package (http://www.aips.nrao.edu/cook.html). In order to calibrate and reduce the data we have used the Common Astronomy Software Application (CASA) software package version 5.1.6 (McMullin et al. 2007), the full documentation can be found at https://casa.nrao.edu/casadocs. The observational parameters for IRAS $06056+2131$ are listed in Table 1 . The observations and data reduction followed normal MERLIN procedures, see the MERLIN User Guide (Diamond et al. 2003).

The $\mathrm{OH}$ maser lines were observed in a spectral bandwidth of $0.5 \mathrm{MHz}$ ('narrow') corresponding to $80 \mathrm{~km} \mathrm{~s}^{-1}$ useful velocity range with a channel separation of $0.18 \mathrm{~km} \mathrm{~s}^{-1}$. The source $3 \mathrm{C} 84$ was observed as a bandpass calibrator in both 
wide and narrow configurations. At this frequency the flux density for $3 \mathrm{C} 84$ was set to be $17.05 \mathrm{Jy}$ based on previous scaling using $3 \mathrm{C} 286$, which has a flux density around $13.6 \mathrm{Jy}$ Baars et al. (1977), allowing for the resolution of MERLIN.

We used 3C84 to correct for the wide-narrow phase offset. The polarization leakage for each antenna was estimated using the un-polarized source $3 \mathrm{C} 84$ while the calibration and correction for the polarization position angle was carried out using the source $3 \mathrm{C} 286$, which has a known polarization angle of $33^{\circ}$ in the image plane. These corrections, along with the bandpass table and the phase reference solutions for phase and amplitude, were applied to the target.

The CLEAN algorithm in CASA package "tclean" was used to clean and de-convolve the image cubes, using the default Cotton-Schwab based deconvolution (Schwab 1984) and the Hogbom minor cycle deconvolver (Högbom 1974). We cleaned all Stokes parameters $I, Q, U$ and $V$ (total intensity, linear and circular products, see Section 3, Equations 1-4), with the same mask, and similarly cleaned the correlator circular polarization products RR and LL. The $\sigma_{\text {rms }}$ noise in quiet channels was $0.03 \mathrm{Jy}$ in $I, Q, U$ and $V$, and $0.04 \mathrm{Jy}$ in RR and LL. The synthesised beam size was $0^{\prime \prime} 19 \times 00^{\prime \prime} 13$ at PA $=$ $29.39^{\circ}$. We made a linearly-polarized intensity (POLI) image using $0.03 \mathrm{Jy}$ for de-biasing, and a polarization angle (POLA) cube, using a cut-off of 3 times the POLI $\sigma_{\text {rms }}$ of $0.04 \mathrm{Jy}$ (see Sect. 3 , Eqs. 5 and 6). Figure 1 displays the brightest channels of IRAS $06056+2131$ after cleaning each circular polarization separately.

In this work we use 'component' to refer to a single patch of emission in a single channel (sometimes referred to as 'spots'), and 'feature' to refer to a series of components which are likely to make up a physical association. We used the CASA task "imfit" to determine the positions of the maser components('spots') by fitting 2D Gaussian components to each patch of emission above a $3 \sigma_{\mathrm{rms}}$ threshold in each channel map for the total intensity (Stokes $I$ ) cube. The $\mathrm{OH}$ maser position errors due to the angular separation and the cycle time between the phase-reference source and the target, are about $0 .{ }^{\prime \prime} 008$ and $0 .{ }^{\prime \prime} 022$, respectively. The stochastic error due to noise for the peak is $0 . \prime 007$. Allowing for the phase reference and telescope position errors, which are comparatively very small, we obtain a total astrometric error of $0 . \prime 025$ in each direction.

The position error for fitting emission imaged using a sparse array such as MERLIN is approximately (synthesised beam)/(signal-to-noise ratio) so the components were required to appear in at least three consecutive channels with positions within 0. . 05 of their occurrence in each consecutive channel, such groups forming spectral features. It was apparent that the resulting components all occurred at the same position within the errors (see Section 3), in the $V_{\mathrm{LSR}}$ range $8.95-10.53 \mathrm{~km} \mathrm{~s}^{-1}$

We therefore measured the flux densities of the other polarization cubes at the same position as the error-weighted Stokes $I$ position. In the case of $Q$, one $2 \sigma_{\text {rms }}$ result is given because the other polarization products are significant. We did this in two ways, firstly by using imfit to fit an unresolved Gaussian component the size of the restoring beam and secondly by using imstat to measure the maximum (or minimum, for $Q, U$ and $V$ ) flux density within the beam area. The results of both methods were the same to within the noise-based positional error.

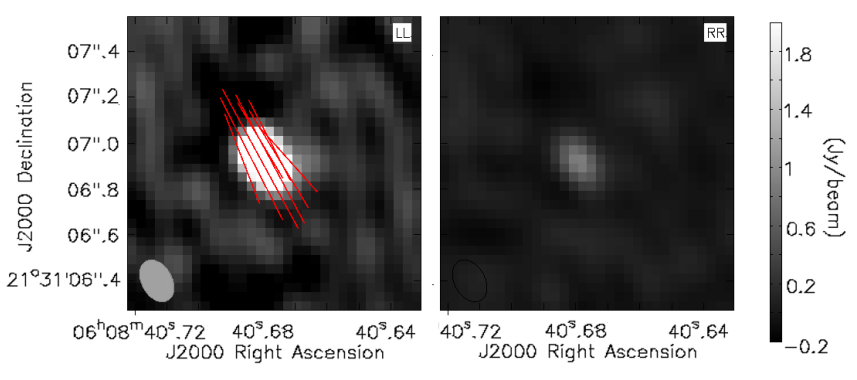

Figure 1. Maps of the clean image of the brightest target channel $\left(V_{\mathrm{LSR}}=10 \mathrm{~km} / \mathrm{s}\right)$. The left panel shows left-hand circular polarization, while the right panel shows right-hand circular polarization. The ellipse in the left corner represents the MERLIN primary beam shape. The intensity is shown by the grey scale while the red lines represent the linear polarization vectors direction.

\section{RESULTS AND DATA ANALYSIS}

We detected $\mathrm{OH}$ maser emission at the $1665-\mathrm{MHz}$ main line toward IRAS $06056+2131$. At the time of our MERLIN observations, the other main line at $1667 \mathrm{MHz}$ and the satellite line at $1720 \mathrm{MHz}$ are absent.

We detected total intensity (Stokes $I$ ) maser components in 9 successive channels (Table 2). The absolute position of the total intensity peak is $\mathrm{RA}=06^{h} 08^{m} 40^{s} .6791, \mathrm{Dec}=21^{\circ}$ $31^{\prime} 6^{\prime \prime} 929$ at $V_{\mathrm{LSR}}=10 \mathrm{~km} \mathrm{~s}^{-1}$. The positions of total intensity components in other channels were close to this, within the noise-based errors. Thus, we adopted the error-weighted centroid of the Stokes $I$ emission as common position for all channels and polarizations, of $\mathrm{RA}=06^{h} 08^{m} 40^{s} .6775$, Dec $=$ $21^{\circ} 31^{\prime} 6^{\prime \prime} \cdot 918$, standard deviations of $0^{\prime \prime} 021$ in RA and $0^{\prime \prime} 017$ in Dec.

Left-hand circular (LHC) polarization masers were detected in 8 of the 9 channels, and right-hand circular (RHC) in 4 channels. The LHC peak coincides with the total intensity peak. Their spectra are shown in Figure 2. If we assume that these are a Zeeman pair then all the $\mathrm{OH} 1665 \mathrm{MHz}$ masers detected comprise a single feature.

In order to measure the Zeeman splitting we fitted spectral Gaussian curves to the LHC and RHC peaks. The peak $V_{\mathrm{LSR}}$ and intensities are given in Table 3. The letter $\mathrm{Z}$ is associated with the RHC and LHC polarized peaks of the identified Zeeman pair. From the identified Zeeman pair, we are able to determine the line-of-sight magnetic field $\left(B_{\|}\right)$in the massive star forming region IRAS $06056+2131$ by measuring the velocity difference between the two polarization hands Elitzur (1996).

We also measured the Stokes parameters $(I, Q, U$ and $V)$, following the radio definitions:

$$
\begin{aligned}
I(u, v) & =1 / 2[\operatorname{RR}(u, v)+\operatorname{LL}(u, v)] \\
V(u, v) & =1 / 2[\operatorname{RR}(u, v)-\operatorname{LL}(u, v)] \\
Q(u, v) & =1 / 2[\operatorname{RL}(u, v)+\operatorname{LR}(u, v)] \\
U(u, v) & =1 / 2 i[\operatorname{LR}(u, v)-\operatorname{RL}(u, v)]
\end{aligned}
$$

$I(u, v)$ is the Stokes integrated (total) flux density, $Q(u, v)$ and $U(u, v)$ are the Stokes flux densities corresponding to the two orthogonal components of linear polarization, while $V(u, v)$ represents the circular component of polarized emission. 
Table 1. IRAS 06056+2131 observational parameters.

\begin{tabular}{cc}
\hline Date of observation & $6,7,8$ and 9 Feb.2007 \\
No. antenna & six $(\mathrm{Cm}, \mathrm{Da}, \mathrm{De}, \mathrm{Kn}, \mathrm{Pi}$ and Mk2) \\
Field centre (RA, Dec J2000) & $06^{h} 08^{m} 40^{s} .97,21^{\circ} 31^{\prime} 00^{\prime \prime} 60$ \\
Rest frequencies (MHz) & 1665.4021667 .3591720 .530 \\
No. of frequency channels & 255 \\
Total band width (MHz) & 0.25 \\
Bandpass calibrator & $3 \mathrm{C} 84$ \\
Polarization angle calibrator & $3 \mathrm{C} 286$ \\
Phase calibrator & $0617+210$ \\
rms in quiet channel (Jy beam & \\
\end{tabular}

Table 2. Measured peak flux densities per channel and errors $\left(\sigma_{\mathrm{rms}}\right)$ in Stokes $I, Q, U, V$, LL, RR, polarized intensity $P$ and polarization angle $\chi$. The symbol - means that no emission was detected above $3 \times$ the given $\sigma_{\text {rms }}$ error.

\begin{tabular}{|c|c|c|c|c|c|c|c|c|c|c|c|c|c|c|c|c|}
\hline \multirow{2}{*}{$\frac{\begin{array}{c}V_{\mathrm{LSR}} \\
\left(\mathrm{km} \mathrm{s}^{-1}\right)\end{array}}{10.53}$} & \multicolumn{2}{|c|}{$\begin{array}{c}I \quad I \text { err } \\
\left(\mathrm{mJy} \mathrm{b}^{-1}\right)\end{array}$} & \multicolumn{2}{|c|}{$\begin{array}{cc}V \quad V \text { err } \\
\left(\mathrm{mJy} \mathrm{b}^{-1}\right)\end{array}$} & \multicolumn{2}{|c|}{ 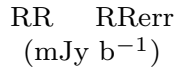 } & \multicolumn{2}{|c|}{$\begin{array}{l}\text { LL LLerr } \\
\left(\mathrm{mJy}^{-1}\right)\end{array}$} & \multicolumn{2}{|c|}{$\begin{array}{cr}Q & Q \text { err } \\
\left(\mathrm{mJy} \mathrm{b}^{-1}\right)\end{array}$} & \multicolumn{2}{|c|}{$\begin{array}{cr}U & U \text { err } \\
\left(\mathrm{mJy} \mathrm{b}^{-1}\right)\end{array}$} & \multicolumn{2}{|c|}{$\begin{array}{cr}P & P \text { err } \\
\left(\mathrm{mJy} \mathrm{b}^{-1}\right)\end{array}$} & \multicolumn{2}{|c|}{$\begin{array}{lr}\chi & \chi \mathrm{err} \\
\text { (degrees) }\end{array}$} \\
\hline & 131 & 19 & -180 & 34 & - & 40 & 298 & 35 & - & 30 & - & 30 & - & - & - & - \\
\hline 10.35 & 127 & 42 & -197 & 42 & - & 40 & 282 & 65 & - & 30 & - & 30 & - & - & - & - \\
\hline 10.18 & 1119 & 48 & -1138 & 51 & - & 40 & 2257 & 89 & - & 30 & - & 30 & - & - & - & - \\
\hline 10.00 & 3808 & 130 & -3673 & 133 & 250 & 58 & 7504 & 250 & 112 & 28 & 197 & 36 & 217 & 33 & 29 & 4 \\
\hline 9.82 & 1702 & 60 & -1665 & 91 & - & 40 & 3375 & 126 & 55 & 30 & 203 & 31 & 205 & 34 & 36 & 4 \\
\hline 9.47 & 102 & 31 & - & 30 & - & 40 & 138 & 55 & - & 30 & - & 30 & - & - & - & - \\
\hline 9.30 & 192 & 39 & 299 & 29 & 476 & 39 & - & 40 & - & 30 & - & 30 & - & - & - & - \\
\hline 9.12 & 545 & 19 & 391 & 35 & 927 & 42 & 164 & 34 & - & 30 & - & 30 & - & - & - & - \\
\hline 8.95 & 230 & 26 & 163 & 38 & 375 & 49 & 251 & 58 & - & 30 & - & 30 & - & - & - & - \\
\hline
\end{tabular}

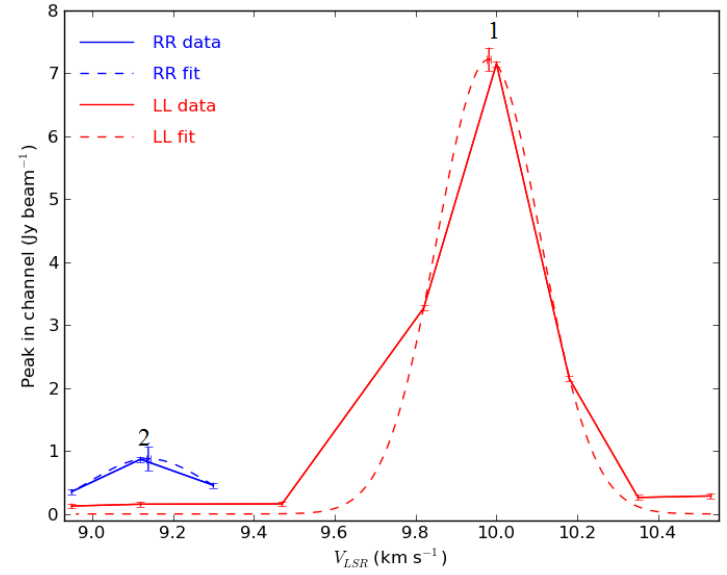

Figure 2. 1665-MHz OH maser spectra towards IRAS 06056+2131 at RA $=06^{h} 08^{m} 40^{s} .6775$, Dec $=21^{\circ} 31^{\prime} 6^{\prime \prime} 918$. The LHC and RHC polarization peaks are labeled No.1 and No.2 respectively. Solid lines are used for the data while dashed lines are for the Gaussian fits.

The degree of linear $\left(P_{\text {linear }}\right)$ and circular $\left(P_{\text {circular }}\right)$ polarization was calculated as:

$$
\begin{aligned}
P_{\text {linear }} & =\left(Q^{2}+U^{2}\right)^{0.5} / I \\
P_{\text {circular }} & =V / I
\end{aligned}
$$

Table 3. Gaussian fit parameters of the RHC and LHC polarization peaks of the $1665-\mathrm{MHz} \mathrm{OH}$ maser detected towards IRAS $06056+2131$.

\begin{tabular}{ccccc}
\hline $\begin{array}{c}\text { Peak } \\
\left(\mathrm{km} \mathrm{s}^{-1}\right)\end{array}$ & $\begin{array}{c}V_{\text {LSR }} \\
(\mathrm{Jy} / \text { beam })\end{array}$ & $\begin{array}{c}\text { Peak Flux } \\
(\mathrm{mG})\end{array}$ & Zeeman Pair & $B_{\|}$ \\
\hline 1. LHC & 9.981 & 7.675 & $\mathrm{Z}$ & -1.5 \\
2. RHC & 9.139 & 0.934 & & \\
\hline
\end{tabular}

The total polarization degree $P_{\text {total }}$ can be then calculated from Eq.7 as:

$P_{\text {total }}=\left(Q^{2}+U^{2}+V^{2}\right)^{0.5} / I$

From Equations 3 and 4, the polarization position angle $\chi$ was calculated as:

$\chi=0.5 \times \arctan (U / Q)$

The Stokes and other polarization parameters were measured from the data cubes as described in Section 2 and listed in Table 2. Note that the POLI cube corresponds to $\left(Q^{2}+U^{2}\right)^{0.5}$ and POLA to $\chi$. Once we determine $P_{\text {linear }}$ and $P_{\text {circular }}$ polarization degrees from equations 5 and 6 respectively, the percentage of those parameters can be identified by $m_{\mathrm{L}}, m_{\mathrm{C}}$ and $m_{\mathrm{T}}$ for the linear, circular and total polarization, respectively and are listed in Table 4. 
Table 4. The polarization angle $(\chi)$ and percentages of linear $\left(\mathrm{m}_{L}\right)$, circular $\left(\mathrm{m}_{C}\right)$ and total $\left(\mathrm{m}_{T}\right)$ polarization, with respect to total intensity, of the $1665-\mathrm{MHz} \mathrm{OH}$ maser emission detected towards IRAS $06056+2131$.

\begin{tabular}{llll}
\hline $\begin{array}{l}m_{\mathrm{L}} \\
(\circ)\end{array}$ & $\begin{array}{l}m_{\mathrm{C}} \\
(\%)\end{array}$ & $\begin{array}{l}m_{\mathrm{T}} \\
(\%)\end{array}$ \\
\hline 29 & 6.0 & 96.5 & 96.6 \\
\hline
\end{tabular}

\section{DISCUSSION}

Our MERLIN OH maser observations represent the first polarimetric study at high spectral and angular resolution of IRAS $06056+2131$. The first detection of $\mathrm{OH}$ maser emission towards this source was made by Turner (1979). They observed the 4 ground-state $\mathrm{OH}$ transitions. Though they tabulated putative detections of $1667 \mathrm{MHz}$ maser emission, the strongest component, at $V_{\mathrm{LSR}}=9.2 \mathrm{~km} \mathrm{~s}^{-1}$, is in the range 3 to 5 sigma according to their peak-to-peak noise values. They nonetheless made a clear detection of the $1665-\mathrm{MHz}$ transition, centred at a velocity of $V_{\mathrm{LSR}}=8.9 \mathrm{~km} \mathrm{~s}^{-1}$ with a full width half maximum of $4.6 \mathrm{~km} \mathrm{~s}^{-1}$ (note that their channel resolution is $1.4 \mathrm{~km} \mathrm{~s}^{-1}$ ).

The detection of $1665-\mathrm{MHz} \mathrm{OH}$ emission, centred at $V_{\mathrm{LSR}}=10 \mathrm{~km} \mathrm{~s}^{-1}$, was then confirmed by Cohen et al. (1988) within $1^{\prime}$ from the IRAS source. They measured a total flux density of $3.4 \mathrm{Jy}$ and $0.3 \mathrm{Jy}$ in the LHC and RHC polarization respectively. Moreover, Edris et al. (2007) using Nançay and Green Bank Telescope observations detected emission at 1667 and $1720 \mathrm{MHz}$ in addition to the $1665 \mathrm{MHz}$ line at velocities $9.44,3.37$ and $10.14 \mathrm{~km} \mathrm{~s}^{-1}$ with flux densities 0.22 , 0.6 and 3.23 Jy respectively. Despite the similarity in velocities of the 1667 and $1665 \mathrm{MHz}$ masers, our non-detection at $1667 \mathrm{MHz}$ could be due to variability (since it was previously much weaker than $1665 \mathrm{MHz}$ ) but could also indicate that the environment is becoming warmer (Cragg et al. 2002; Gray et al. 1991).

Previous surveys of the $1720 \mathrm{MHz} \mathrm{OH}$ maser line by Fish et al. (2003) and Ruiz-Velasco et al. (2016) suggested that this line is clearly observed from regions with relatively high magnetic field strengths which coincide with $\mathrm{H}$ II regions. The significant difference in the velocity between the $1720-\mathrm{MHz}$ line detected by Edris et al. (2007) and the main lines could be an indication that the $1720-\mathrm{MHz}$ is tracing another protostellar object or that it is excited by different phenomena.

\subsection{Polarization}

The derived polarization parameters listed in Table 4 indicate that IRAS $06056+2131$ is highly circularly polarized source. However, at the velocity of the LHC peak we found a linear polarization percentage of $6 \%$ and a circular polarization percentage of $96.5 \%$, which means that the emission is elliptically polarized. The line-of-sight component of the magnetic field at the location of the Zeeman pair is $B_{\|}=-1.5 \mathrm{mG}$, indicating a magnetic field pointing toward us. Figure 3 shows the detected $\mathrm{OH}$ maser emission through different channels of IRAS $06056+2131$ with their circular polarization contours and linear polarization vectors. Figure 4 shows the total intensity (Stokes $I$ ) of IRAS $06056+2131$, where we can see clearly the Zeeman pair. It also displays the measured linear polarization intensity labeled with their polarization angles. The contribution of the magnetic field in the plane of the sky, which is perpendicular to the polarisation vectors could suggest that it is broadly aligned with the possible SE-NW CO outflow detected by Xu et al. (2006), see Section 4.2 and Figure 5. However, the foreground Faraday rotation can potentially be strong at low frequencies hence making it harder to interpret the magnetic field direction (e.g. Vlemmings et al. 2006a).

In order to estimate this effect on the orientation of the measured polarization position angle $(\chi)$, we used the same method as in Etoka \& Diamond (2010) relying on the measurement of the dispersion measure (DM) and the rotation measure (RM) from pulsars. The closest pulsar to IRAS $06056+2131$ is found to be PSR J0609+2130. This pulsar is located at $1.2 \mathrm{kpc}$ and has a dispersion measure (DM) of $38.77 \mathrm{~cm}^{-3}$ pc (Lorimer et al. 2004). Unfortunately, no $\mathrm{RM}$ is provided for this pulsar. The next closest pulsar with both a known DM of $96.91 \mathrm{~cm}^{-3} \mathrm{pc}$ and a known RM of $66.0 \mathrm{rad} \mathrm{m}^{2}$, PSR B0611+22, is located with an offset of $1.6^{\circ}$ from IRAS $06056+2131$ at a comparable distance of $1.74 \mathrm{kpc}$ (Sobey, priv. communication).

Assuming that the ambient Galactic magnetic field at the location of PSR J0609+2130 and PSR B0611+22 is similar, the RM for PSR J0609+2130 is then inferred to be $26.4 \mathrm{rad} \mathrm{m}^{2}$, based on Eq. 1 from Han et al. (1999), given by:

$B_{\|}=1.232(\mathrm{RM} / \mathrm{DM})$

Since the degree of Faraday rotation is given by $\mathrm{RM} \lambda^{2}$ (Noutsos et al. 2008), this implies that the values of $\chi$ measured here potentially suffer from Faraday rotation as high as $50^{\circ}$.

Additionally, internal Faraday rotation can also affect the linear polarisation information if the $\mathrm{OH}$ masers are located right behind the UCHII region. Using Fish \& Reid (2006) eq. 5 and the electron density in addition to the diameter of the masing region from Kurtz et al. (1994) (cf. their Table 5), would lead to a very high value of internal Faraday rotation. On the other hand, Fish \& Reid (2006) show that even if there is a substantial amount of Faraday rotation (i.e., $>1$ $\mathrm{rad}$ ) along the amplification length, the key length is the gain length. The fact that we measure a significant percentage of linear polarisation implies that internal Faraday rotation potentially affecting the measurements of $\chi$ along the gain length is $<1 \mathrm{rad}$ (i.e., $<57^{\circ}$ ), suggesting that the masers arise from the limb or the near side of the UC H II region. Since the sense of the external and internal potential Faraday rotation is nonetheless not known, the measurements of $\chi$ can suffer from $0^{\circ}$ to up to $110^{\circ}$ of rotation.

The alignment of the polarisation vectors with the orientation of the $\mathrm{CO}$ outflow can consequently be fortuitous. It is to be noted though, that the velocity of the masers is in good agreement with their suggested association with the red lobe of the $\mathrm{CO}$ outflow.

We performed a rough comparison between the energy density in the magnetic field of strength $B$ and the kinetic energy density in the CO outflow (see also Section 4.2) in order to investigate whether the magnetic field could have a significant effect, following the method summarised in Assaf et al. (2013). We assume that $B=B_{\|}$; if $B$ is greater then the magnetic energy density will be even greater. The magnetic energy density is given by $E_{\mathrm{B}}=B^{2} /\left(2 \mu_{0}\right)$ where $\mu_{0}$ 


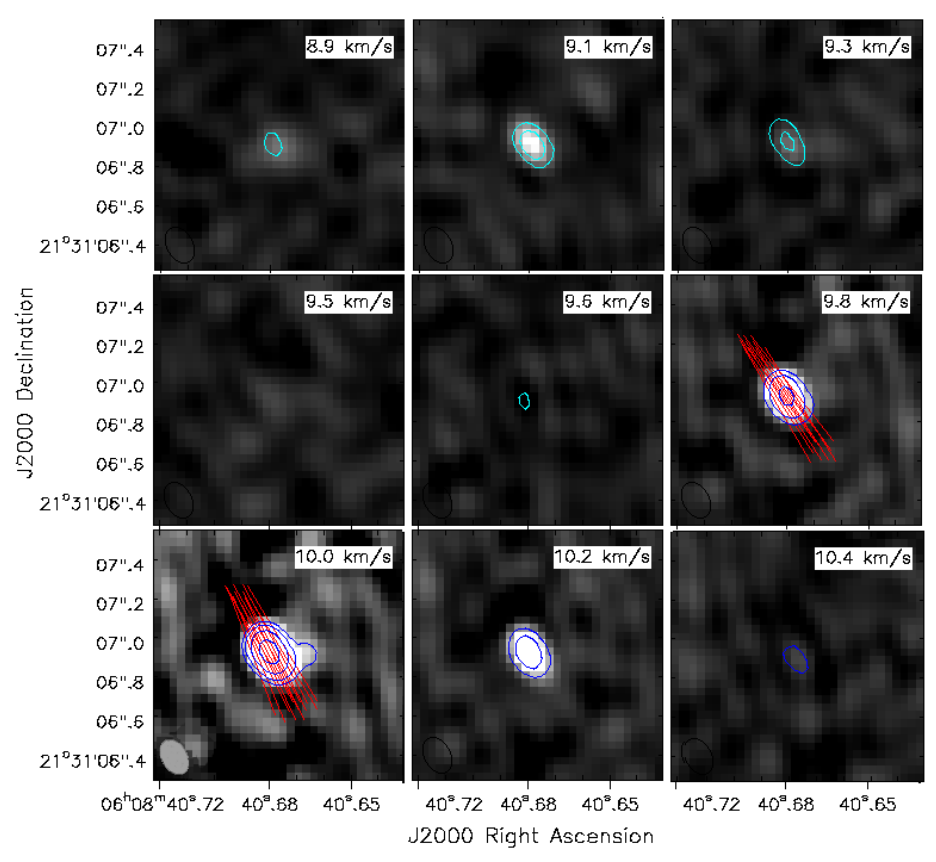

Figure 3. Channel maps of IRAS $06056+2131$. The contour levels are adapted to the dynamic range per panel. The grey scale shows the total intensity, cut at $[-0.1,1]$ Jy. The light blue contours show Stokes $V$ at $(1,2) \times 12 \mathrm{mJy} /$ beam. The dark blue contours show Stokes $V$ at $(1,2,4,8) \times-32 \mathrm{mJy} /$ beam in the first panels, $-12 \mathrm{mJy} /$ beam $\left(3 \sigma_{\mathrm{rms}}\right)$ in the last panel (at $\left.V_{\mathrm{LSR}}=10.4 \mathrm{~km} \mathrm{~s}^{-1}\right)$. The filled beam is shown at lower left of the panel at $V_{\mathrm{LSR}}=10.0 \mathrm{~km} \mathrm{~s}^{-1}$. The red vectors represent the linear polarization position angle where the linear polarization is significant (at a threshold of $3 \sigma_{\mathrm{rms}}$ ). See Table 2 for details.

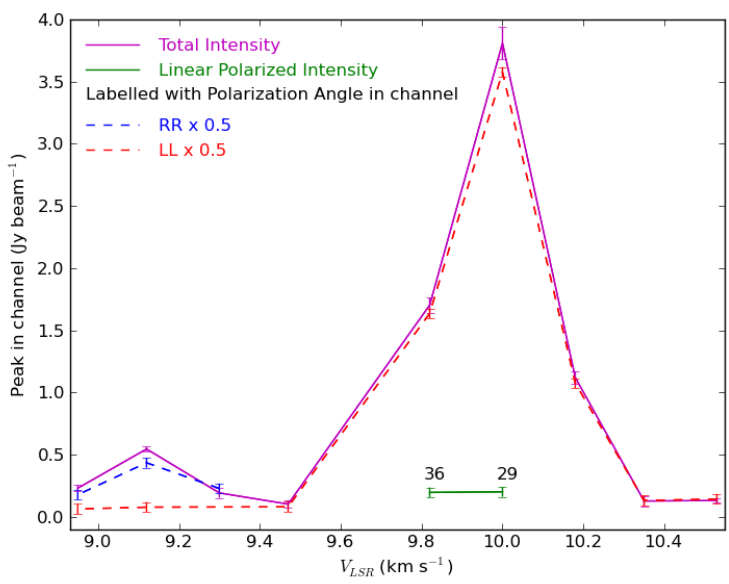

Figure 4. The total and polarized intensity spectra of $1665 \mathrm{MHz}$ toward IRAS $06056+2131$. The green solid bar is labeled with the linear polarization angle per channel. RR and LL have been multiplied by 0.5 to facilitate comparison with Stokes $I$ and provide a more compact plot. is the permeability of free space. For $B$ in Gauss, this gives $E_{\mathrm{B}} \approx 0.004 B^{2} \approx 9 \times 10^{-9} \mathrm{~J} \mathrm{~m}^{-3}$.

The thermal energy density is given by $E_{\mathrm{th}}=3 / 2 n k_{\mathrm{B}} T_{\mathrm{K}}$ where $k_{\mathrm{B}}$ is Boltzmann's constant. The non-detection of the $1667 \mathrm{GHz}$ maser implies that the gas temperature $T_{\mathrm{K}}>75$ $\mathrm{K}$ and the number density $n>10^{10} \mathrm{~m}^{-3}$ (Cragg et al. 2002; Gray et al. 1991). This leads to $E_{\mathrm{th}}>1.5 \times 10^{-11} \mathrm{~J} \mathrm{~m}^{-3}$. Even for a much higher temperature of $200 \mathrm{~K}, E_{\mathrm{th}}<E_{\mathrm{B}}$ as long as $n<2 \times 10^{12} \mathrm{~m}^{-3}$.

The bulk energy density is given by $E_{\mathrm{Bulk}}=0.5 \rho v^{2}$ where we assume the velocity $v$ as the $\mathrm{CO}$ outflow velocity of 7.5 $\mathrm{km} \mathrm{s}^{-1}$, half the maximum velocity span measured by $\mathrm{Xu}$ et al. (2006). The gas density $\rho=n \times 1.25 m_{\mathrm{H}_{2}}$, where $m_{\mathrm{H}_{2}}$ is the mass of the hydrogen molecule. Thus, $E_{\mathrm{Bulk}}<E_{\mathrm{B}}$ for $n<8 \times 10^{10} \mathrm{~m}^{-3}$.

These estimates are very crude but show that the magnetic field is likely to influence the outflow for number densities in the range $1-8 \times 10^{10} \mathrm{~m}^{-3}\left(1-8 \times 10^{7} \mathrm{~cm}^{-3}\right)$ within a temperature range of $75-200 \mathrm{~K}$.

\subsection{The evolutionary status}

To understand the evolutionary status of the star forming region IRAS $06056+2131$, we put together all the results of the different observations with known position performed close to the $\mathrm{OH}$ maser. This is presented in Figure 5. This figure has the MERLIN OH maser position, RA $=06^{h} 08^{m} 40^{s} .6775$, Dec $=21^{\circ} 31^{\prime} 6^{\prime \prime} .918$ at the origin, marked by a blue cross the size of which represents the position error $\left(0 .{ }^{\prime \prime} 025\right)$.

We estimated the positions of the $\mathrm{OH}$ masers observed using the Greenbank Telescope (GBT) using maps provided by (Edris et al. 2007). The $1665-\mathrm{MHz}$ LHC maser peak at $10.14 \mathrm{~km} \mathrm{~s}^{-1}$, is at RA $=06^{h} 08^{m} 41^{s} .095$, Dec $=21^{\circ} 31^{\prime}$ $21^{\prime \prime} .21$, uncertainty 38 arcsec. This is offset by $(5.8,14.3)$ arcsec from the MERLIN position, within the GBT uncertainty. However, the $1720 \mathrm{MHz}$ maser peak, at $3.4 \mathrm{~km} \mathrm{~s}^{-1}$, is at $\mathrm{RA}=06^{h} 08^{m} 44^{s} .348$, Dec $=21^{\circ} 31^{\prime} 40^{\prime \prime}$.01, uncertainty 40 arcsec, an offset of $(51,33)$ arcsec. The highest-resolution observation of the $6.7 \mathrm{GHz}$ methanol maser was made by $\mathrm{Xu}$ et al. (2009) using MERLIN, with $\sim 30$ mas astrometric accuracy (based on analysis of the original data) at a peak position of RA $=06^{h} 08^{m} 40^{s} .671$, Dec $=21^{\circ} 31^{\prime} 06^{\prime \prime} .89$, velocity $8.8 \mathrm{~km} \mathrm{~s}^{-1}$. This is marked by the magenta diamond ( $\sim 10$ times larger than the position error) in Figure 5 . In a recent observation, $\mathrm{Hu}$ et al. (2016) detected the maser at the same position within uncertainties, peaking at $9.39 \mathrm{~km} \mathrm{~s}^{-1}$.

Koempe et al. (1989), using Effelsberg, found that the 22$\mathrm{GHz} \mathrm{H}_{2} \mathrm{O}$ maser is offset by $-20^{\prime \prime}, 8^{\prime \prime}$ from the IRAS source, position accuracy $10^{\prime \prime}$, at a velocity $2 \mathrm{~km} \mathrm{~s}^{-1}$, shown by the cyan circle and error bars in Figure 5.

Fontani et al. (2010) using the Nobeyama 45-m telescope failed to detect any Class-I methanol maser emission lines (44 $\mathrm{GHz}$ and $95 \mathrm{GHz}$ ) towards IRAS $06056+2131$, On the other hand, their Effelsberg observations of the Class-II $6.7 \mathrm{GHz}$ methanol maser transition show a complex spectrum with emission in the entire velocity range $[8.5 ; 11] \mathrm{km} \mathrm{s}^{-1}$, with at least 3 spectral components, the strongest being at $8.8 \mathrm{~km} \mathrm{~s}^{-1}$ with peak flux density $=12.7 \mathrm{Jy}$.

The most precise radio continuum observation of the UC H II region was made by $\mathrm{Hu}$ et al. (2016) using the VLA, giving a position of $\mathrm{RA}=06^{h} 08^{m} 40^{s} .67, \mathrm{Dec}=21^{\circ} 31^{\prime} 07^{\prime \prime} .2$. The astrometry is accurate to at least $0 . \prime 3$. The extent of the 


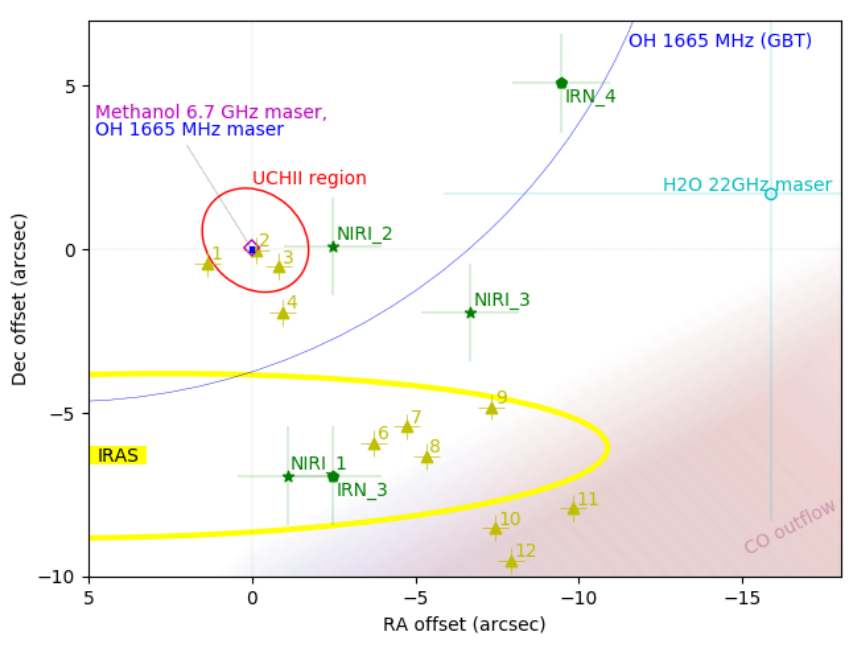

Figure 5. Overview of the star-forming region IRAS $06056+2131$. The origin of coordinates $(0,0)$ is the location of the $\mathrm{OH}$ maser peak at $\mathrm{RA}=06^{h} 08^{m} 40^{s} .6775$, Dec $=21^{\circ} 31^{\prime} 06^{\prime \prime} \cdot 918$, shown by the blue cross. The error circle of the $1665-\mathrm{MHz}$ observation by Edris et al. (2007) is shown by the blue arc. The magenta diamond and cyan circle mark the methanol (Xu et al. 2009) and water (Koempe et al. 1989) masers, respectively. The red ellipse marks the UC H II region ( $\mathrm{Hu}$ et al. 2016). The dark green star symbols mark the IR sources identified by Kwon et al. (2018), while the light green triangle symbols labeled from 1 to 12 represent the Smithsonian Millimetre Array (SMA) detections by Rodón et al. (2012). The yellow ellipse marks the IRAS source position bounds, and the shaded area marks an estimate of the $\mathrm{CO}$ outflow direction from $\mathrm{Xu}$ et al. (2006). See Section 4 for details.

UCH II region is shown by the red ellipse (with angular size of $3^{\prime \prime} .5 \times 22^{\prime \prime} 9$ at position angle $41.4^{\circ}$ ) in Figure 5 . The position of IRAS $06056+2131$ is RA $=06^{h} 08^{m} 40^{s} .973$, Dec $=21^{\circ} 31^{\prime}$ $00^{\prime \prime} 61$, with an error ellipse of axes $(30,5)$ arcsec with the long axis almost exactly E-W (Joint IRAS Science Working Group 1988)(shown in yellow in Figure 5), is likely to represent an aggregate of sources within the IRAS beam. Several of the Infra-red Reflection Nebulae (IRN) and Near Infra-Red Illuminators (NIRI) identified by Kwon et al. (2018) (cf. their Tables 1 and 2) are within 10 arcsec of the $\mathrm{OH}$ maser. We assumed that the astrometric accuracy was typical for the detector used, probably $\sim 1$ 1. 5 ( $\sim 3$ pixels $)$. These sources are shown and labeled in dark green in Figure 5 . Note the existence of a WISE source, at RA $=06^{h} 08^{m} 40^{s} .45$, Dec $=$ $21^{\circ} 31^{\prime} 02^{\prime \prime}$, , which is within the positional uncertainty of the IRAS source (Kwon et al. 2018). Consequently, as noted by Kwon et al. (2018) the WISE source probably corresponds to the IRAS source and is not shown separately in Figure 5.

Rodón et al. (2012) detected $12 \mathrm{~mm}$-wave sources in the region, using the SMA. We assumed that the published positions (estimated from their figure 1) were relative to the pointing position $\mathrm{RA}=06^{h} 08^{m} 40^{s} .31$, Dec $=21^{\circ} 31^{\prime} 03^{\prime \prime} .6$ listed in the SMA (Smithsonian Millimetre Array) archive, with an astrometric accuracy of 0.4 (1/3 of a synthesised beam). These sources are shown and numbered in light green in Figure 5.

Figure 5 shows that the hot core SMA 2, the $\mathrm{OH} 1665 \mathrm{MHz}$ maser and the methanol maser are closely associated. SMA 2 is approximately 0.130 arcsec west of the $\mathrm{OH}$ maser, within the position error. The methanol maser is $(0.095 \pm 0.039)$ arc- sec from the $\mathrm{OH}$ maser, which corresponds to $\sim 140 \pm 60 \mathrm{au}$ in the plane of the sky at assumed distance of $1.5 \mathrm{kpc}$. Such a small separation between the $\mathrm{OH}$ and Class II methanol masers indicates that they are from a very similar region at overlapping velocities (around $8-10 \quad \mathrm{~km} \mathrm{~s}^{-1}$ ). These all lie in the direction of the UCHII region, in fact SMA 2 is the closest hot core to the region centre.

The close association (including similar velocities) between $\mathrm{OH}$ and Class II methanol masers is likely to indicate a relatively later evolutionary stage of massive star formation than regions which only have methanol masers (e.g Caswell 1997; Breen et al. 2010a).

The water maser is offset from these masers by much more than the combined errors and peaks at a lower velocity $(\sim$ $\left.2 \mathrm{~km} \mathrm{~s}^{-1}\right)$. The position and the velocity of the water maser suggests that it might be pumped by shocks associated with the $\mathrm{CO}$ outflow.

The pale red-blue shaded strip represents the orientation of the CO outflow mapped by Xu et al. (2006) from their figure 1, along the SE - NW axis joining the blue- and red-shifted peaks. It should be noted that $\mathrm{CO}$ emission was detected throughout the area and the association of these peaks with the same outflow is tentative.

Zhang et al. (2005), using the NRAO $12 \mathrm{~m}$ telescope detected a $\mathrm{CO} \mathrm{J}=2-1$ outflow which they associated with a deeply embedded IR source and the UCHII region. These are located at an offset of $\left(-14^{\prime \prime} .0,14^{\prime \prime} .0\right)$ in RA and Dec from the IRAS source (see figure 2 of Zhang et al. 2005). Xu et al. (2006) carried out higher resolution imaging of CO J=2-1 using the Nobeyama Radio Telescope. The integrated emission peak is at $2.5 \mathrm{~km} \mathrm{~s}^{-1}$ and two pairs of red and blue-shifted peaks are resolved, covering a total velocity range of about - 5 to $+10 \mathrm{~km} \mathrm{~s}^{-1}$. The CO outflow appears to be oriented close to the line of sight although Kwon et al. (2018) point out that at high resolution the region is complex and other interpretations are possible. The $\mathrm{OH}$ maser velocity appears to be close to the most red-shifted CO velocity of $10 \mathrm{~km} \mathrm{~s}^{-1}$ and the polarization vectors (which are perpendicular to the magnetic field lines), suggest that the magnetic field orientation might be in agreement with that of the SE-NW CO outflow reported by $\mathrm{Xu}$ et al. (2006) (see their figure 1). The direction indicated by $B_{\|}$suggests that the magnetic field associated with the far outflow points towards the observer. In addition to the outflow, Yoo et al. (2018) reported evidence for infalling material, using observations of lines of $\mathrm{HCO}^{+}$ $(1-0), \mathrm{HCO}^{+}(3-2), \mathrm{H}_{2} \mathrm{CO}\left(2_{12}-1_{11}\right)$ and $\mathrm{H}^{13} \mathrm{CO}^{+}(1-0)$.

The presence of both outflow and infall material could be additional evidence for a protostellar object in an early evolutionary stage, when the radiation force is still lower than the gravitational force at the outer boundary.

The observations conducted toward IRAS 06056+2131 suggest that it is a high-mass star-forming region at a not so early evolutionary phase, associated with an UCH II region detected by Koempe et al. (1989); Kurtz et al. (1994) and at highest resolution by $\mathrm{Hu}$ et al. (2016).

\subsection{Comparison with other sources}

Main-line OH masers towards the IRAS sources 20126+4104 and 19092+0841 (hereafter IRAS 20126, 19092) were also imaged, using MERLIN, at a similar angular resolution to this study, by Edris et al. (2005) and Edris et al. (2007), respec- 
tively. The velocity range of the $\mathrm{OH}$ maser emission at 1665 $\mathrm{MHz}$ detected towards them is 17 and $3 \mathrm{~km} \mathrm{~s}^{-1}$, respectively.

IRAS 20126 is at $1.7 \mathrm{kpc}$ (Wilking et al. 1989), a similar distance as our target IRAS $06065+2131$, which has a velocity range of the maser emission at $1665-\mathrm{MHz}$ of $1.5 \mathrm{~km} \mathrm{~s}^{-1}$. In IRAS 20126, the two brightest $\mathrm{OH}$ maser features at $1665 \mathrm{MHz}$ (peak fluxes > 0.9Jy), which are separated by $14 \mathrm{~km} \mathrm{~s}^{-1}$, are so bright that they would have been detected with MERLIN toward both IRAS 19092 (D=4.48 kpc, Molinari et al. 1996) and IRAS 06056+2131. However, these two sources show an intrinsically smaller $V_{\mathrm{LSR}}$ span than IRAS 20126.

Compared with IRAS sources 20126 and 19092 (with extents of 2000 and $22400 \mathrm{au}$, respectively), the $\mathrm{OH}$ maser is very compact in IRAS $06056+2131$ with an upper limit to its distribution of 0.03 arcsec which corresponds to 45 au at an assumed distance of $1.5 \mathrm{kpc}$. Both $\mathrm{OH}$ maser main lines were detected toward IRAS 19092 while only $1665 \mathrm{MHz}$ was found toward IRAS 20126 and IRAS 06056+2131, suggesting a higher gas temperature and density for the latter two sources (Gray et al. 1991).

There is no evidence for $\mathrm{OH}$ masers tracing a circumstellar disk either in IRAS $06056+2131$ or IRAS 19092, while Edris et al. (2005) reported that the $\mathrm{OH}$ masers are tracing a circumstellar disk in IRAS 20126. The small upper limit to the size of the OH maser towards IRAS 06056+2131 makes it improbable that the small observed $V_{\mathrm{LSR}}$ range is a projection effect of an outflow in the plane of the sky.

IRAS $06056+2131$ is relatively less luminous in comparison with IRAS 20126 and IRAS $19092\left(5.83 \times 10^{3} \mathrm{~L}_{\odot}, 10^{4} \mathrm{~L}_{\odot}\right.$ and $10^{4} \mathrm{~L} \odot$, respectively) while the line-of-sight magnetic field measured from the $\mathrm{OH}$ Zeeman pair has the lowest magnitude (1.5, 11 and $4.4 \mathrm{mG}$, respectively). The $6.7 \mathrm{GHz}$ methanol (Class II) maser has been detected toward the three IRAS sources, while class I has been detected only toward IRAS 19092.

No radio-continuum was detected towards IRAS 20126 (Molinari et al. 1998) and Edris et al. (2017) showed that the nearest detection is offset by about 2 arcmin from IRAS 19092, suggesting that the $\mathrm{OH}$ masers in these sources are not associated with UCHII regions, unlike the presence of a UC H II region associated with IRAS $06056+2131$.

Bearing in mind all the results summarised above, we suggest that IRAS $06056+2131$ is at an evolutionary stage comparable with IRAS 20126, which is more evolved than IRAS 19092. This result is consistent with what was reported by Edris et al. (2007), where they found the sources with higher $\mathrm{OH}$ intensity to be more evolved. The absence of any Class I methanol maser from IRAS 06056+2131 is a further evidence that it is more evolved than IRAS 19092 (Ellingsen 2006).

\section{CONCLUSIONS}

We have presented a high angular resolution observations of $\mathrm{OH}$ maser emission toward IRAS $0605+2131$. At the time of the observation of the three $\mathrm{OH}$ transitions at 1665, 1667 and $1720 \mathrm{MHz}$, only $1665 \mathrm{MHz}$ was detected. The small upper limit to the size of the $\mathrm{OH}$ maser emitting region is estimated to be $\sim 45$ au at $1.5 \mathrm{kpc}$. We measured the line-of-sight magnetic field from the identified $\mathrm{OH}$ Zeeman pair to be $\sim-1.5$ $\mathrm{mG}$, and the corresponsing magnetic field energy density is strong enough to influence the outflow. The linear polarization vectors might suggest that the magnetic field orientation in the plane of the sky is roughly NW to SE. This might then be aligned with the possible $\mathrm{CO}$ outflow direction (Xu et al. 2006). However, Faraday rotation gives an uncertainty of $110^{\circ}$ in the actual magnetic field direction.

Our results are found to be in a good agreement with Caswell (1998), which tested the association between $\mathrm{OH}$ and 6.7-GHz masers with a large sample and concluded that, $80 \%$ of $\mathrm{OH}$ masers have $6.7-\mathrm{GHz}$ methanol maser counterparts. The spatial and velocity coincidence between $\mathrm{OH}$ and Class II methanol maser indicates that they are probably tracing the same physical phenomena in IRAS 06056+2131, however there is no evidence of a circumstellar disk. The close association of a Class-II methanol maser and UCHII region with the $\mathrm{OH} 1665-\mathrm{MHz}$ maser and the absence of ClassI methanol masers suggest that IRAS $06056+2131$ is at a later stage of evolution than sources without detectable $\mathrm{OH}$ masers. In comparison with other $\mathrm{OH}$ maser sources investigated by Edris et al. (2005) and Edris et al. (2017), the properties of IRAS $06056+2131$ also suggests that is at a relatively more evolved evolutionary stage.

Finally, higher angular resolution would provide a better estimate of the location and properties of the protostellar object driving the IRAS $06056+2131$ outflow, for example VLBI measurements of masers and ALMA measurements of the $\mathrm{mm} / \mathrm{sub}-\mathrm{mm}$ core.

\section{ACKNOWLEDGEMENTS}

We gratefully thank Prof. R. Battye, M. Gray and R. Beswick, and the rest of the e-MERLIN team for guidance in reducing these data. We also remember the important role of the late Dr Jim Cohen in initiating this project. We thank the anonymous referee for very insightful and helpful comments which have improved this paper. e-MERLIN is the UK radio interferometer array, operated by the University of Manchester on behalf of STFC. We acknowledge the use of MERLIN archival data as well as NASA's Astrophysics Data System Service. M.Darwish would like to acknowledge the Science and Technology Development Fund (STDF) N5217, Academy of Scientific Research and Technology (ASRT), Cairo, Egypt and Kottamia Center of Scientific Excellence for Astronomy and Space Sciences (KCSEASSc), National Research Institute of Astronomy and Geophysics (NRIAG). Our sincere thanks to S. Ellingsen and J. Allotey for their helpful discussion and to C. Sobey for pulsar data.

\section{DATA AVAILABILITY}

The raw visibility data and the clean images cubes in FITS format for all $\mathrm{OH}$ maser polarization products of the MERLIN observations of IRAS $06056+2131$ can be found at: https://doi.org/10.5281/zenodo.3961902.

\section{REFERENCES}

Assaf K. A., Diamond P. J., Richards A. M. S., Gray M. D., 2013, MNRAS, 431, 1077 
Baars J. W. M., Genzel R., Pauliny-Toth I. I. K., Witzel A., 1977, A\&A, 61, 99

Braz M. A., Lepine J. . R. D., Sivagnanam P., Le Squeren A. M. ., 1990, A\&A, 236, 479

Breen S. L., Ellingsen S. P., Caswell J. L., Lewis B. E., 2010a, MNRAS, 401, 2219

Breen S. L., Caswell J. L., Ellingsen S. P., Phillips C. J., 2010b, MNRAS, 406, 1487

Bronfman L., Nyman L. A., May J., 1996, A\&AS, 115, 81

Caswell J. L., 1997, MNRAS, 289, 203

Caswell J. L., 1998, MNRAS, 297, 215

Caswell J. L., Vaile R. A., Ellingsen S. P., Whiteoak J. B., Norris R. P., 1995, MNRAS, 272, 96

Caswell J. L., Kramer B. H., Reynolds J. E., 2011, MNRAS, 415, 3872

Cohen R. J., 1985, in Kahn F. D., ed., Cosmical Gas Dynamics. pp 223-235

Cohen R. J., Baart E. E., Jonas J. L., 1988, MNRAS, 231, 205

Commerçon B., Hennebelle P., Henning T., 2011, ApJ, 742, L9

Cragg D. M., Sobolev A. M., Godfrey P. D., 2002, MNRAS, 331, 521

Crutcher R. M., Kemball A. J., 2019, Frontiers in Astronomy and Space Sciences, 6, 66

Darwish M. S., Edris K. A., Richards A. M. S., Etoka S., Saad M. S., Beheary M. M., Fuller G. A., 2020, MNRAS, 493, 4442

Diamond P. J., Garrington S. T., Gunn A. G., Leahy J. P., McDonald A., Muxlow T. W. B., Richards A. M. S., Thomasson P., 2003, Technical report, The MERLIN User Guide. Jodrell Bank Observatory, UK., http://www.merlin.ac.uk/user_guide/MUG.ps.gz

Edris K. A., Fuller G. A., Cohen R. J., Etoka S., 2005, A\&A, 434, 213

Edris K. A., Fuller G. A., Cohen R. J., 2007, A\&A, 465, 865

Edris K. A., Fuller G. A., Etoka S., Cohen R. J., 2017, A\&A, 608, A 80

Elitzur M., 1996, ApJ, 457, 415

Ellingsen S. P., 2006, ApJ, 638, 241

Ellingsen S. P., Voronkov M. A., Cragg D. M., Sobolev A. M., Breen S. L., Godfrey P. D., 2007, in Chapman J. M., Baan W. A., eds, IAU Symposium Vol. 242, Astrophysical Masers and their Environments. pp 213-217 (arXiv:0705.2906), doi:10.1017/S1743921307012999

Etoka S., Diamond P. J., 2010, MNRAS, 406, 2218

Etoka S., Gray M. D., Fuller G. A., 2012, MNRAS, 423, 647

Fish V. L., Reid M. J., 2006, ApJS, 164, 99

Fish V. L., Reid M. J., 2007, ApJ, 670, 1159

Fish V. L., Reid M. J., Argon A. L., Menten K. M., 2003, ApJ, 596,328

Fontani F., Cesaroni R., Furuya R. S., 2010, A\&A, 517, A56

Garay G., Lizano S., 1999, PASP, 111, 1049

Goddi C., Surcis G., Moscadelli L., Imai H., Vlemmings W. H. T., van Langevelde H. J., Sanna A., 2017, A\&A, 597, A43

Gray M. D., Doel R. C., Field D., 1991, MNRAS, 252, 30

Green J. A., Richards A. M. S., Vlemmings W. H. T., Diamond P., Cohen R. J., 2007, MNRAS, 382, 770

Green J. A., McClure-Griffiths N. M., Caswell J. L., Robishaw T., Harvey-Smith L., 2012, Monthly Notices of the Royal Astronomical Society, 425, 2530

Han J. L., Manchester R. N., Qiao G. J., 1999, MNRAS, 306, 371

Högbom J. A., 1974, A\&AS, 15, 417

Hu B., Menten K. M., Wu Y., Bartkiewicz A., Rygl K., Reid M. J., Urquhart J. S., Zheng X., 2016, ApJ, 833, 18

Joint IRAS Science Working Group 1988, Infrared Astronomical Satellite (IRAS) Catalogs and Atlases: The Point source catalog declination range $30^{\circ}>\delta>0^{\circ}$. Vol. 1190, Scientific and Technical Information Division, National Aeronautics and ...

Kitsionas S., Jappsen A. K., Klessen R. S., Whitworth A. P., 2005, in Protostars and Planets V Posters. p. 8555
Klassen M., Pudritz R. E., Kirk H., 2017, MNRAS, 465, 2254

Koempe C., Baudry A., Joncas G., Wouterloot J. G. A., 1989, A\&A, 221, 295

Kurtz S., Churchwell E., Wood D. O. S., 1994, ApJS, 91, 659

Kwon J., et al., 2018, AJ, 156, 1

Lorimer D. R., et al., 2004, MNRAS, 347, L21

Mac Low M.-M., Klessen R. S., 2004, Reviews of Modern Physics, 76,125

McKee C. F., Tan J. C., 2003, ApJ, 585, 850

McMullin J. P., Waters B., Schiebel D., Young W., Golap K., 2007, in Shaw R. A., Hill F., Bell D. J., eds, Astronomical Society of the Pacific Conference Series Vol. 376, Astronomical Data Analysis Software and Systems XVI. p. 127

Molinari S., Brand J., Cesaroni R., Palla F., 1996, A\&A, 308, 573

Molinari S., Brand J., Cesaroni R., Palla F., Palumbo G. G. C., 1998, A\&A, 336, 339

Momjian E., Sarma A. P., 2017, ApJ, 834, 168

Mouschovias T. C., Paleologou E. V., 1979, ApJ, 230, 204

Mouschovias T. C., Tassis K., Kunz M. W., 2006, ApJ, 646, 1043

Noutsos A., Johnston S., Kramer M., Karastergiou A., 2008, MNRAS, 386, 1881

Padoan P., Nordlund Å., 2002, ApJ, 576, 870

Palla F., Brand J., Cesaroni R., Comoretto G., Felli M., 1991, A\&A, 246, 249

Rodón J. A., Beuther H., Schilke P., Zhang Q., 2012, Boletin de la Asociacion Argentina de Astronomia La Plata Argentina, 55, 199

Rosolowsky E., et al., 2010, ApJS, 188, 123

Ruiz-Velasco A. E., Felli D., Migenes V., Wiggins B. K., 2016, ApJ, 822,101

Schwab F. R., 1984, AJ, 89, 1076

Snell R. L., Huang Y. L., Dickman R. L., Claussen M. J., 1988, ApJ, 325, 853

Stahler S. W., Palla F., Ho P. T. P., 2000, in Mannings V., Boss A. P., Russell S. S., eds, Protostars and Planets IV. pp 327-352

Sunada K., Nakazato T., Ikeda N., Hongo S., Kitamura Y., Yang J., 2007, PASJ, 59, 1185

Surcis G., Vlemmings W. H. T., Curiel S., Hutawarakorn Kramer B., Torrelles J. M., Sarma A. P., 2011a, A\&A, 527, A48

Surcis G., Vlemmings W. H. T., Torres R. M., van Langevelde H. J., Hutawarakorn Kramer B., 2011b, A\&A, 533, A47

Surcis G., Vlemmings W. H. T., van Langevelde H. J., Hutawarakorn Kramer B., Quiroga-Nuñez L. H., 2013, A\&A, 556, A73

Szymczak M., Gérard E., 2004, A\&A, 414, 235

Szymczak M., Hrynek G., Kus A. J., 2000, A\&AS, 143, 269

Tan J. C., Kong S., Butler M. J., Caselli P., Fontani F., 2013, ApJ, 779,96

Turner B. E., 1979, A\&AS, 37, 1

Vázquez-Semadeni E., Banerjee R., Gómez G. C., Hennebelle P., Duffin D., Klessen R. S., 2011, MNRAS, 414, 2511

Vlemmings W. H. T., 2007, in Chapman J. M., Baan W. A., eds, IAU Symposium Vol. 242, Astrophysical Masers and their Environments. pp 37-46 (arXiv:0705.0885), doi:10.1017/S1743921307012549

Vlemmings W. H. T., Harvey-Smith L., Cohen R. J., 2006a, MNRAS, 371, L26

Vlemmings W. H. T., Diamond P. J., van Langevelde H. J., Torrelles J. M., 2006b, A\&A, 448, 597

Vlemmings W. H. T., Surcis G., Torstensson K. J. E., van Langevelde H. J., 2010, MNRAS, 404, 134

Wilking B. A., Mundy L. G., Blackwell J. H., Howe J. E., 1989, ApJ, 345, 257

Wood D. O. S., Churchwell E., 1989, ApJ, 340, 265

Wu Y., Wei Y., Zhao M., Shi Y., Yu W., Qin S., Huang M., 2004, A\&A, 426, 503

Xu Y., et al., 2006, AJ, 132, 20 
Xu Y., Voronkov M. A., Pandian J. D., Li J. J., Sobolev A. M., Brunthaler A., Ritter B., Menten K. M., 2009, A\&A, 507, 1117 Yoo H., Kim K.-T., Cho J., Choi M., Wu J., Evans Neal J. I., Ziurys L. M., 2018, ApJS, 235, 31

Zhang Q., Hunter T. R., Brand J., Sridharan T. K., Cesaroni R., Molinari S., Wang J., Kramer M., 2005, ApJ, 625, 864

This paper has been typeset from a $\mathrm{T}_{\mathrm{EX}} / \mathrm{LATEX}$ file prepared by the author. 